\title{
A modified F-expansion method for solving nonlinear pseudo- parabolic BBMB equations
}

\author{
${ }^{1}$ Priyanka M. Patel and ${ }^{2}$ Vikas H. Pradhan \\ ${ }^{1,2}$ Department of applied mathematics, S. V. National Institute of Technology, Surat-395007, India
}

\begin{abstract}
In this paper, the modified F-expansion method with the aid of symbolic computational system Mathematica is implemented to obtain many exact travelling wave solutions for a special form of generalized nonlinear pseudo-parabolic Benjamin-Bona-Mohany-Burgers (BBMB) equation. The obtained solutions are expressed by the hyperbolic functions and the trigonometric functions. This method presents a wider applicability for handling nonlinear pseudo-parabolic partial differential equations arising from the mathematical physics.
\end{abstract}

Keywords:generalizednonlinear pseudo-parabolic Benjamin-Bona-Mohany-Burgers equation, modified Fexpansion method,travelling wave solutions

\section{Introduction}

In recent years, nonlinear partial differential equations (NLPDEs) have been used to model many physical phenomena in various fields of science and engineering. Thus, it is important to investigate the exact solutions of NLPDEs. To find the exact solutions of NLPDEs many methods have been developed such as variational iteration method [1], homotopy perturbation method [2], tanh function method [3], $\left(G^{\prime} / G\right)$ expansion method [4], exp-function method [5] and many others. In the recent past, a straight-forward and concise method was proposed to construct travelling wave solutions of NLPDEs, called F-expansion method $[6,7,8]$. This method was later further extended in different manners.

In this paper, we used a modified F-expansion method $[9,10]$ to obtain exact travelling wave solutions of the generalized BBMBequation [11],

$$
u_{t}+\beta u_{x}-\alpha u_{x x}+(g(u))_{x}-u_{x x t}=0
$$

where $u(x, t)$ represents the velocity of fluid in the horizontal direction $x . \alpha$ is the positive constant. $\beta \in R$ and $g(u)$ is a $C^{2}$-smooth nonlinear function. With the dissipative term $\alpha u_{x x}$ eq. (1) arises in the phenomena for both the bore propagation and the water waves. Eq.(1) with $\alpha=0, \beta=1$ and $g(u)_{x}=u u_{x}$ converted into Benjamin-Bona-Mohany (BBM) equation proposed by Peregrine [12] and Benjamin, Bona and Mohany [13]. Eq.(1) has been handled by many authors using different mathematical techniques such as El-wakil et al. [11] used the exp-function method to obtain the generalized solitary and periodic solutions for eq.(1) by considering $g(u)=\frac{u^{2}}{2}$. In [14] Al-khaled et al. obtained approximate solutions of eq.(1) by using adomain decomposition method. Fakhari et al. [15] implemented the homotopy analysis method to eq.(1) with $\alpha=0, \beta=1$ and $g(u)=\frac{u^{2}}{2}$. Tari and Ganji [16] applied variational iteration method andhomotopy perturbation method to derive approximate explicit solutions of eq.(1).Gözükizil and Akçağilsolved eq.(1) by using tanh method [17].

The aim of this work is to obtain new exact solitary-like and periodic solutions of generalized BBMB equation with $g(u)=u u_{x}$ and $g(u)=\frac{u^{2}}{2}$ and also to describe the efficiency of the method to handle the nonlinear pseudo- parabolic type partial differential equations.

The outline of this paper is as follows: In section 2, we give the basic idea of the modified F-expansion method. In section 3, we apply this method to generalized BBMB equation. In section 4, some conclusions are given. 


\section{Basic idea of modified F-expansion method}

Consider a given nonlinear partial differential equation with two independent variables $x, t$ and dependent variable $u$.

$$
P\left(u, u_{t}, u_{x}, u_{x t}, u_{x x}, u_{t t}, \ldots\right)=0
$$

where, $P$ is a polynomial in $u$ and its various partial derivatives.

The main points for solving eq.(2) are as follows:

[1]. Assume the travelling wave solutions of eq.(2) in the form

$$
u(x, t)=u(\xi) \text { where } \xi=h(x+w t)
$$

where $h \neq 0$ and $w$ are constants to be determined. Substituting eq.(3) into eq.(2), eq.(2) becomes an ordinary differential equation (ODE)

$$
P\left(u, u^{\prime}, u^{\prime \prime}, \ldots\right)=0
$$

where prime denotes the derivative with respect to $\xi$.

[2]. Suppose that the solution of eq.(4) can be expressed in the finite series form

$$
u(\xi)=\sum_{i=-N}^{N} a_{i} F^{i}(\xi) \text { where } a_{N} \neq 0
$$

where $a_{i}(i=-N, \ldots,-1,0,1, \ldots, N)$ are all constants to be determined and $F(\xi)$ satisfies following Riccati equation

$$
F^{\prime}(\xi)=A+B F(\xi)+C F^{2}(\xi)
$$

where $A, B, C$ are constants and $N$ is a positive integer which can be determined by balancing the orders of nonlinear term with the higher order derivative term in eq.(4). Also

(i) when $N=(p / q)$ is fraction, let $u(\xi)=v^{p / q}(\xi)$

(ii) when $N$ is negative integer, let $u(\xi)=v^{N}(\xi)$

By using (i) and (ii), we change eq.(4) into another ODE for $v(\xi)$, whose balancing number will be a positive integer.

[3]. Substituting eq.(5) together with eq.(6) into eq.(4) then the left-hand side of the eq.(4) can be converted into finite series in $F^{p}(\xi),(p=-N, \ldots,-1,0,1, \ldots, N)$. Equating each coefficient of $F^{p}(\xi)$ to zero gives a system of algebraic equations for $a_{i}, h, w$.

[4]. Solve the system of algebraic equations with the aid of computer symbolic system like Mathematica or Maple, we can obtain the constants $a_{i}, h, w$. Substituting these results into eq.(5), we can obtain the general form of travelling wave solutions to eq.(4).

[5]. From the general form of travelling wave solutions of eq.(6) listed in appendix, we can give series of soliton-like solutions and trigonometric function solutions of eq.(2).

\section{Application to generalized BBMB equation}

Case 1.Consider $g(u)=u u_{x}$ in eq.(1), we get

$$
u_{t}+\beta u_{x}-\alpha u_{x x}+u_{x}^{2}+u u_{x x}-u_{x x t}=0
$$

We assume that eq.(7) has travelling wave solution in the form

$$
u(x, t)=u(\xi) \text { where } \xi=h(x+w t)
$$

where $h \neq 0, w$ are constants. Substituting eq.(8) into eq.(7), we get following ordinary differential equation,

$$
(\beta+w) u^{\prime}-\alpha h u^{\prime \prime}+h\left(u u^{\prime}\right)^{\prime}-h^{2} w u^{\prime \prime \prime}=0
$$

where prime denotes differentiation with respect to $\xi$. Integrating eq. (9) and considering integration constant is zero. We have

$$
(\beta+w) u-\alpha h u^{\prime}+h\left(u u^{\prime}\right)-h^{2} w u^{\prime \prime}=0
$$


Now, balancing the orders of $u u^{\prime}$ and $u^{\prime \prime}$ in eq.(10), we get, $N=1$. So we can write solution of eq.(7) in the following form,

$$
u(x, t)=u(\xi) \text { where } u(\xi)=a_{0}+a_{-1} F^{-1}(\xi)+a_{1} F(\xi)
$$

where $a_{0}, a_{-1}, a_{1}$ are constants to be determined later. Substituting eq.(11) into eq.(10) and using eq.(6), the left hand side of eq.(10) can be converted into a finite series in $F^{p}(\xi),(p=-3,-2,-1,0,1,2,3)$, equating each coefficient of $F^{p}(\xi)$ to zero, we find the system of algebraic equations for $a_{0}, a_{-1}, a_{1}, h, w$.

$$
\left.\begin{array}{l}
F^{-3}(\xi):-A h a_{-1}{ }^{2}-2 A^{2} h^{2} a_{-1} w=0 \\
F^{-2}(\xi):-A h a_{0} a_{-1}-B h a_{-1}{ }^{2}-3 A B h^{2} a_{-1} w+A h a_{-1} \alpha=0 \\
F^{-1}(\xi):-B h a_{0} a_{-1}-C h a_{-1}{ }^{2}+a_{-1} w-B^{2} h^{2} a_{-1} w-2 A C h^{2} a_{-1} w+B h a_{-1} \alpha+a_{-1} \beta=0 \\
F^{0}(\xi):-C h a_{0} a_{-1}+A h a_{0} a_{1}+a_{0} w-B C h^{2} a_{-1} w-A B h^{2} a_{1} w+C h a_{-1} \alpha-A h a_{1} \alpha+a_{0} \beta=0 \\
F^{1}(\xi): B h a_{0} a_{1}+A h a_{1}^{2}+a_{1} w-B^{2} h^{2} a_{1} w-2 A C h^{2} a_{1} w-B h a_{1} \alpha+a_{1} \beta=0 \\
F^{2}(\xi): C h a_{0} a_{1}+B h a_{1}^{2}-3 B C h^{2} a_{1} w-C h a_{1} \alpha=0 \\
F^{3}(\xi): C h a_{1}^{2}-2 C^{2} h^{2} a_{1} w=0
\end{array}\right\}
$$

Solving the above algebraic equations with the aid of Mathematica, we obtain following values of $a_{0}, a_{-1}, a_{1}, w$

When $A=0$, we have

$$
a_{0}=\alpha-B h \beta, a_{-1}=0, a_{1}=-2 C h \beta, w=-\beta
$$

When $B=0$, we have

$$
a_{0}=\alpha, a_{-1}=0, a_{1}=-2 C h \beta, w=-\beta
$$

where $\mathrm{h}$ is left as a free parameter.

Substituting values of $a_{0}, a_{-1}, a_{1}, w$ into eq.(11) and using appendix, we can obtain many soliton-like solutions and trigonometric function solutions of eq.(7).

(1) Select $A=0, B=1, C=-1$ and $F(\xi)=\frac{1}{2}+\frac{1}{2} \tanh \left(\frac{\xi}{2}\right)$ from appendix and using eq.(13), we have,

$$
u_{1}(x, t)=\alpha+h \beta \tanh \left[\frac{h(x-\beta t)}{2}\right]
$$

(2) Select $A=0, B=-1, C=1$ and $F(\xi)=\frac{1}{2}-\frac{1}{2} \operatorname{coth}\left(\frac{\xi}{2}\right)$ from appendix and using eq.(13), we have,

$$
u_{2}(x, t)=\alpha-h \beta \operatorname{coth}\left[\frac{h(x-\beta t)}{2}\right]
$$

(3) Select $A=\frac{1}{2}, B=0, C=-\frac{1}{2}$ and $F(\xi)=\operatorname{coth}(\xi) \pm \operatorname{csch}(\xi), \tanh (\xi) \pm i \sec h(\xi)$ from appendix and using eq.(14), we have,

$$
\begin{aligned}
& u_{3}(x, t)=\alpha+h \beta(\operatorname{coth}[h(x-\beta t)] \pm \csc h[h(x-\beta t)]) \\
& u_{4}(x, t)=\alpha+h \beta(\tanh [h(x-\beta t)] \pm i \sec h[h(x-\beta t)])
\end{aligned}
$$

(4) Select $A=1, B=0, C=-1$ and $F(\xi)=\tanh (\xi), \operatorname{coth}(\xi)$ from appendix and using eq.(14), we have,

$$
u_{5}(x, t)=\alpha+2 h \beta(\tanh [h(x-\beta t)])
$$




$$
u_{6}(x, t)=\alpha+2 h \beta(\operatorname{coth}[h(x-\beta t)])
$$

(5) Select $A=\frac{1}{2}, B=0, C=\frac{1}{2}$ and $F(\xi)=\sec (\xi)+\tan (\xi), \csc (\xi)-\cot (\xi)$ from appendix and using eq.(14), we have,

$$
\begin{aligned}
& u_{7}(x, t)=\alpha-h \beta(\sec (h(x-\beta t))+\tan (h(x-\beta t))) \\
& u_{8}(x, t)=\alpha-h \beta(\csc (h(x-\beta t))-\cot (h(x-\beta t)))
\end{aligned}
$$

(6) Select $A=-\frac{1}{2}, B=0, C=-\frac{1}{2}$ and $F(\xi)=\sec (\xi)-\tan (\xi), \csc (\xi)+\cot (\xi)$ from appendix and using eq.(14), we have,

$$
\begin{aligned}
& u_{9}(x, t)=\alpha+h \beta(\sec (h(x-\beta t))-\tan (h(x-\beta t))) \\
& u_{10}(x, t)=\alpha+h \beta(\csc (h(x-\beta t))+\cot (h(x-\beta t)))
\end{aligned}
$$

(7) Select $A=1, B=0, C=1$ and $F(\xi)=\tan (\xi)$ from appendix and using eq.(14), we have,

$$
u_{11}(x, t)=\alpha-2 h \beta \tan [h(x-\beta t)]
$$

(8) Select $A=-1, B=0, C=-1$ and $F(\xi)=\cot (\xi)$ from appendix and using eq.(14), we have,

$$
u_{12}(x, t)=\alpha+2 h \beta \cot [h(x-\beta t)]
$$

Case 2. Taking $g(u)=\frac{u^{2}}{2}$ in eq.(1), we get

$$
u_{t}+\beta u_{x}-\alpha u_{x x}+u u_{x}-u_{x x t}=0
$$

We assume that eq.(27) has travelling wave solution in the form

$$
u(x, t)=u(\xi) \text { where } \xi=h(x+w t)
$$

where $h \neq 0, w$ are constants. Substituting eq.(28) into eq.(27), we get ordinary differential equation,

$$
(\beta+w) u-\alpha h u^{\prime}+\frac{u^{2}}{2}-h^{2} w u^{\prime \prime}=0
$$

Balancing the orders of $u^{2}$ and $u^{\prime \prime}$ in eq.(29), we get $N=2$. So we may choose,

$$
u(\xi)=a_{0}+a_{-2} F^{-2}(\xi)+a_{-1} F^{-1}(\xi)+a_{1} F^{1}(\xi)+a_{2} F^{2}(\xi)
$$

where $a_{0}, a_{-2}, a_{-1}, a_{1}, a_{2}$ are constants to be determined later. Substituting eq.(30) into eq.(29) and using eq.(6), the left hand side of eq.(29) can be converted into a finite series in $F^{p}(\xi)$, $(p=-4,-3,-2,-1,0,1,2,3,4)$, equating each coefficient of $F^{p}(\xi)$ to zero, we find the system of algebraic equations for $a_{-2}, a_{-1}, a_{0}, a_{1}, a_{2}, h, w$. 


$$
\begin{aligned}
& F^{-4}(\xi): \frac{a_{-2}^{2}}{2}-6 A^{2} h^{2} a_{-2} w=0 \\
& F^{-3}(\xi): a_{-2} a_{-1}+2 A h a_{-2} \alpha-10 A B h^{2} a_{-2} w-2 A^{2} h^{2} a_{-1} w=0 \\
& F^{-2}(\xi): a_{0} a_{-2}+\frac{a_{-1}^{2}}{2}+a_{-2} w+2 B h a_{-2} \alpha+A h a_{-1} \alpha-4 B^{2} h^{2} a_{-2} w-8 A C h^{2} a_{-2} w \\
& -3 A B h^{2} a_{-1} w+a_{-2} \beta=0 \\
& F^{-1}(\xi): a_{-1} \beta+a_{0} a_{-1}+a_{-2} a_{1}+a_{-1} w+2 C h a_{-2} \alpha+B h a_{-1} \alpha-6 B C h^{2} a_{-2} w-B^{2} h^{2} a_{-1} w \\
& -2 A C h^{2} a_{-1} w=0 \\
& \left.F^{0}(\xi): a_{0} \beta+\frac{a_{0}^{2}}{2}+a_{2} a_{-2}+a_{-1} a_{1}+a_{0} w+C h a_{-1} \alpha-A h a_{1} \alpha-2 A^{2} h^{2} a_{2} w-2 C^{2} h^{2} a_{-2} w\right\} \\
& -B C h^{2} a_{-1} w-A B h^{2} a_{1} w=0 \\
& F^{1}(\xi): a_{2} a_{-1}+a_{1} \beta+a_{0} a_{1}+a_{1} w-2 A h a_{2} \alpha-B h a_{1} \alpha-6 A B h^{2} a_{2} w-B^{2} h^{2} a_{1} w \\
& -2 A C h^{2} a_{1} w=0 \\
& F^{2}(\xi): a_{2} \beta+a_{2} a_{0}+\frac{1}{2} a_{1}^{2}+a_{2} w-2 B h a_{2} \alpha-C h a_{1} \alpha-4 B^{2} h^{2} a_{2} w-8 A C h^{2} a_{2} w \\
& -3 B C h^{2} a_{1} w=0 \\
& F^{3}(\xi): a_{2} a_{1}-2 C h a_{2} \alpha-10 B C h^{2} a_{2} w-2 C^{2} h^{2} a_{1} w=0 \\
& F^{4}(\xi): \frac{1}{2} a_{2}^{2}-6 C^{2} h^{2} a_{2} w=0
\end{aligned}
$$

Solving above algebraic equations with the help of Mathematica, we obtain values of $a_{0}, a_{-2}, a_{-1}, a_{1}, a_{2}, h, w$.

Consider $A=0$, we get

$$
\begin{aligned}
& a_{0}=0, a_{-2}=0, a_{-1}=0, a_{1}=0, a_{2}=-\frac{12 C^{2} h \alpha}{5 B}, w=-\frac{\alpha}{5 B h}, \\
& h=\frac{5 \beta \pm \sqrt{25 \beta^{2}-24 \alpha^{2}}}{12 B \alpha} \\
& a_{0}=\frac{2 \alpha-10 B h \beta}{5 B h}, a_{-2}=0, a_{-1}=0, a_{1}=0, a_{2}=-\frac{12 C^{2} h \alpha}{5 B}, w=-\frac{\alpha}{5 B h}, \\
& h=\frac{-5 \beta \pm \sqrt{25 \beta^{2}+24 \alpha^{2}}}{12 B \alpha}
\end{aligned}
$$

Taking $B=0$, we have

$$
\begin{aligned}
& a_{0}=\frac{i\left(1-12 A C h^{2}\right) \alpha}{10 \sqrt{A} \sqrt{C} h}-\beta, a_{-2}=0, a_{-1}=0, a_{1}=\frac{12 C h \alpha}{5}, a_{2}=-\frac{6 i C^{3 / 2} h \alpha}{5 \sqrt{A}}, \\
& w=-i \frac{\alpha}{10 \sqrt{A} \sqrt{C} h}, h=\frac{-5 i \sqrt{A} \sqrt{C} \beta \pm \sqrt{-24 A C \alpha^{2}-25 A C \beta^{2}}}{24 A C \alpha} \\
& \text { or } h=\frac{5 i \sqrt{A} \sqrt{C} \beta \pm \sqrt{24 A C \alpha^{2}-25 A C \beta^{2}}}{24 A C \alpha}
\end{aligned}
$$

Substituting above values of $a_{i}(i=0,-2,-1,1,2), h, w$ in eq.(30) and using appendix, we obtained exact travelling wave of solutions of eq.(27). 
(1) Choose $A=0, B=1, C=-1$ and $F(\xi)=\frac{1}{2}+\frac{1}{2} \tanh \left(\frac{\xi}{2}\right)$ from appendix and using eq.(32), we get,

$$
u_{1}(x, t)=-\frac{3 h \alpha}{5}\left(1+\tanh \left[\frac{h}{2}\left(x-\frac{\alpha}{5 h} t\right)\right]\right)^{2}
$$

Using eq.(33) we have,

$$
u_{2}(x, t)=\frac{2 \alpha-10 h \beta}{5 h}-\frac{3 h \alpha}{5}\left(1+\tanh \left[\frac{h}{2}\left(x-\frac{\alpha}{5 h} t\right)\right]\right)^{2}
$$

(2) Choose $A=0, B=-1, C=1$ and $F(\xi)=\frac{1}{2}-\frac{1}{2} \operatorname{coth}\left(\frac{\xi}{2}\right)$ from appendix and using eq.(32), we get,

$$
u_{3}(x, t)=\frac{3 h \alpha}{5}\left(1-\operatorname{coth}\left[\frac{h}{2}\left(x+\frac{\alpha}{5 h} t\right)\right]\right)^{2}
$$

Using eq.(33) we get

$$
u_{4}(x, t)=\frac{-2 \alpha-10 h \beta}{5 h}+\frac{3 h \alpha}{5}\left(1-\operatorname{coth}\left[\frac{h}{2}\left(x+\frac{\alpha}{5 h} t\right)\right]\right)^{2}
$$

(3) Choose $A=\frac{1}{2}, B=0, C=-\frac{1}{2}$ and $F(\xi)=\operatorname{coth}(\xi) \pm \operatorname{csch}(\xi), \tanh (\xi) \pm i \sec h(\xi)$ from appendix and using eq.(34), we get,

$$
\begin{aligned}
u_{5}(x, t) & =\frac{1}{5}\left(\frac{\alpha}{h}+3 h \alpha-5 \beta\right)-\frac{6 h \alpha}{5}\left(\operatorname{coth}\left[h\left(x-\frac{\alpha}{5 h} t\right)\right] \pm \operatorname{csch}\left[h\left(x-\frac{\alpha}{5 h} t\right)\right]\right) \\
& -\frac{3 h \alpha}{5}\left(\operatorname{coth}\left[h\left(x-\frac{\alpha}{5 h} t\right)\right] \pm \operatorname{csch}\left[h\left(x-\frac{\alpha}{5 h} t\right)\right]\right)^{2} \\
u_{6}(x, t) & =\frac{1}{5}\left(\frac{\alpha}{h}+3 h \alpha-5 \beta\right)-\frac{6 h \alpha}{5}\left(\tanh \left[h\left(x-\frac{\alpha}{5 h} t\right)\right] \pm i \sec h\left[h\left(x-\frac{\alpha}{5 h} t\right)\right]\right) \\
& -\frac{3 h \alpha}{5}\left(\tanh \left[h\left(x-\frac{\alpha}{5 h} t\right)\right] \pm i \sec h\left[h\left(x-\frac{\alpha}{5 h} t\right)\right]\right)^{2}
\end{aligned}
$$

(4) Choose $A=1, B=0, C=-1$ and $F(\xi)=\tanh (\xi), \operatorname{coth}(\xi)$ from appendix and using eq.(34), we get,

$$
\begin{aligned}
& u_{7}(x, t)=\frac{\left(1+12 h^{2}\right) \alpha}{10 h}-\beta-\frac{12 h \alpha}{5} \tanh \left[h\left(x-\frac{\alpha}{10 h} t\right)\right]-\frac{6 h \alpha}{5} \tanh ^{2}\left[h\left(x-\frac{\alpha}{10 h} t\right)\right] \\
& u_{8}(x, t)=\frac{\left(1+12 h^{2}\right) \alpha}{10 h}-\beta-\frac{12 h \alpha}{5} \operatorname{coth}\left[h\left(x-\frac{\alpha}{10 h} t\right)\right]-\frac{6 h \alpha}{5} \operatorname{coth}^{2}\left[h\left(x-\frac{\alpha}{10 h} t\right)\right]
\end{aligned}
$$

(5) Choose $A=\frac{1}{2}, B=0, C=\frac{1}{2}$ and $F(\xi)=\sec (\xi)+\tan (\xi), \csc (\xi)-\cot (\xi)$ from appendix and using eq.(34), we get,

$$
\begin{aligned}
u_{9}(x, t) & =\frac{i\left(1-3 h^{2}\right) \alpha}{5 h}-\beta+\frac{6 h \alpha}{5}\left(\sec \left[h\left(x-i \frac{\alpha}{5 h} t\right)\right]+\tan \left[h\left(x-i \frac{\alpha}{5 h} t\right)\right]\right) \\
& -i \frac{3 h \alpha}{5}\left(\sec \left[h\left(x-i \frac{\alpha}{5 h} t\right)\right]+\tan \left[h\left(x-i \frac{\alpha}{5 h} t\right)\right]\right)^{2}
\end{aligned}
$$




$$
\begin{aligned}
u_{10}(x, t) & =\frac{i\left(1-3 h^{2}\right) \alpha}{5 h}-\beta+\frac{6 h \alpha}{5}\left(\csc \left[h\left(x-i \frac{\alpha}{5 h} t\right)\right]-\cot \left[h\left(x-i \frac{\alpha}{5 h} t\right)\right]\right) \\
& -i \frac{3 h \alpha}{5}\left(\csc \left[h\left(x-i \frac{\alpha}{5 h} t\right)\right]-\cot \left[h\left(x-i \frac{\alpha}{5 h} t\right)\right]\right)^{2}
\end{aligned}
$$

(6) Choose $A=-\frac{1}{2}, B=0, C=-\frac{1}{2}$ and $F(\xi)=\sec (\xi)-\tan (\xi), \csc (\xi)+\cot (\xi)$ from appendix and using eq.(34), we get,

$$
\begin{aligned}
u_{11}(x, t) & =-\frac{i\left(1-3 h^{2}\right) \alpha}{5 h}-\beta-\frac{6 h \alpha}{5}\left(\sec \left[h\left(x+i \frac{\alpha}{5 h} t\right)\right]-\tan \left[h\left(x+i \frac{\alpha}{5 h} t\right)\right]\right) \\
& +i \frac{3 h \alpha}{5}\left(\sec \left[h\left(x+i \frac{\alpha}{5 h} t\right)\right]-\tan \left[h\left(x+i \frac{\alpha}{5 h} t\right)\right]\right)^{2} \\
u_{12}(x, t) & =-\frac{i\left(1-3 h^{2}\right) \alpha}{5 h}-\beta-\frac{6 h \alpha}{5}\left(\csc \left[h\left(x+i \frac{\alpha}{5 h} t\right)\right]+\cot \left[h\left(x+i \frac{\alpha}{5 h} t\right)\right]\right) \\
& +i \frac{3 h \alpha}{5}\left(\csc \left[h\left(x+i \frac{\alpha}{5 h} t\right)\right]+\cot \left[h\left(x+i \frac{\alpha}{5 h} t\right)\right]\right)^{2}
\end{aligned}
$$

(7) Choose $A=1, B=0, C=1$ and $F(\xi)=\tan (\xi)$ from appendix and using eq.(34), we get,

$$
\begin{aligned}
u_{13}(x, t)= & \frac{i\left(1-12 h^{2}\right) \alpha}{10 h}-\beta+\frac{12 h \alpha}{5} \tan \left[h\left(x-i \frac{\alpha}{10 h} t\right)\right] \\
& -i \frac{6 h \alpha}{5} \tan \left[h\left(x-i \frac{\alpha}{10 h} t\right)\right]^{2}
\end{aligned}
$$

(8) Choose $A=-1, B=0, C=-1$ and $F(\xi)=\cot (\xi)$ from appendix and using eq.(34), we get,

$$
\begin{aligned}
u_{14}(x, t)= & -\frac{i\left(1-12 h^{2}\right) \alpha}{10 h}-\beta-\frac{12 h \alpha}{5} \cot \left[h\left(x+i \frac{\alpha}{10 h} t\right)\right] \\
& +i \frac{6 h \alpha}{5} \cot \left[h\left(x+i \frac{\alpha}{10 h} t\right)\right]^{2}
\end{aligned}
$$

\section{Conclusion}

The modified F-expansion method issuccessfully used to obtain travelling wave solutions for nonlinear pseudo-parabolic BBMB equations. Using this method we obtained some hyperbolic functions and trigonometric function solutions which are not seen before. Also obtained solutions satisfy BBMB equations. On the other hand, we have seen that the modified F-expansion method is easy to apply and reliable to solve the pseudo-parabolic partial differential equations.

Appendix: Relations between values of $(A, B, C)$ and corresponding $F(\xi)$ in Riccati equation

$$
F^{\prime}(\xi)=A+B F(\xi)+C F^{2}(\xi)
$$

\begin{tabular}{|c|c|c|c|}
\hline$A$ & $B$ & $C$ & $F(\xi)$ \\
\hline 0 & 1 & -1 & $\frac{1}{2}+\frac{1}{2} \tanh \left(\frac{\xi}{2}\right)$ \\
\hline
\end{tabular}




\begin{tabular}{|c|c|c|c|}
\hline 0 & -1 & 1 & $\frac{1}{2}-\frac{1}{2} \operatorname{coth}\left(\frac{\xi}{2}\right)$ \\
\hline$\frac{1}{2}$ & 0 & $-\frac{1}{2}$ & $\operatorname{coth}(\xi) \pm \operatorname{csch}(\xi), \tanh (\xi) \pm i \sec (\xi)$ \\
\hline 1 & 0 & -1 & $\tanh (\xi), \operatorname{coth}(\xi)$ \\
\hline$\frac{1}{2}$ & 0 & $\frac{1}{2}$ & $\sec (\xi)+\tan (\xi), \csc (\xi)-\cot (\xi)$ \\
\hline$-\frac{1}{2}$ & 0 & $-\frac{1}{2}$ & $\sec (\xi)-\tan (\xi), \csc (\xi)+\cot (\xi)$ \\
\hline $1(-1)$ & 0 & $1(-1)$ & $\tan (\xi), \cot (\xi)$ \\
\hline
\end{tabular}

\section{References}

[1] J-H He, "Variational iteration method- a kind of non-linear analytical technique: some examples ", International journal of nonlinear mechanics, vol. 34, pp. 699-708, 1999

[2] D. D. Ganji, “ The application of He's homotopy perturbation method to nonlinear equations arising in heat transfer ", Physics letters A, vol. 355, pp. 337-341, 2006

[3] W. Malfliet, " The tanh method: a tool for solving certain classes of nonlinear evolution and wave equations", Journal of computational and applied mathematics, vol. 164-165, pp. 529-541, 2004

[4] L. Ling-Xiao, W. Ming-Liang, “The $\left(G^{\prime} / G\right)$ - expansion method and travelling wave solutions for a higher-order nonlinear Schrödinger equation”, Applied mathematics and computation, vol.208, pp. 440-445, 2009

[5] J-H. He, X-H. Wu, “Exp-function method for nonlinear wave equations”, Chaos, solitons\& fractals, vol. 30, pp. 700-708, 2006

[6] M-L. Wang, Y-B. Zhou, "The periodic wave solutions for the Klein-Gordon- Schrodinger equations", Physics letters A, vol. 318, pp. 84-92, 2003

[7] Y. Zhou, M. Wang and Y. Wang, "Periodic wave solutions to a coupled Kdv equations with variable coefficients", Physics letters A, vol. 308, pp. 31-36, 2003

[8] Y-M. Zhao, "F-expansion method and its application for finding new exact solutions to the Kudryashov-sinelshchikov equation", journal of applied mathematics, vol. 2013, pp. 01-07, 2013

[9] G. Cai and Q. Wang, "A modified F-expansion method for solving nonlinear pdes", Journal of information and computing science, vol.2, pp. 03-16, 2007

[10] G. Cai, F. Zhang, Y. Wang "Some new solutions for the nonlinear dispersive-dissipative equation with a modified F-expansion method", World Journal of Modeling and Simulation, vol. 3, pp. 212-219, 2007

[11] S. A. El-Wakil, M. A. Abdou, A. Hendi, “New periodic wave solutions via exp-function method”, Physics letters A, vol. 372, pp. $830-840,2008$

[12] D. H. Peregrine, "Calculations of the development of an undular bore", Journal of fluid mechanics, vol. 25, pp. 321-330, 1966

[13] T. B. Benjamin, J. L. Bona and J. J. Mahony, “ Model equations for long waves in nonlinear dispersive systems”, Philosophical transactions of the royal society A, vol. 272, pp. 47-78, 1972

[14] K. Al-Khaled, S. Momani, A. Alawneh, "Approximate wave solutions for generalized Benjamin-Bona-Mahony-Burgers equations", Applied mathematics and computation, vol. 171, pp. 281-292, 2005

[15] A. Fakhari, G. Domairry, Ebrahimpour, "Approximate explicit solutions of nonlinear BBMB equations by homotopy analysis method and comparison with the exact solution”, Physics letters A, vol. 368, pp. 64-68, 2007

[16] H. Tari, D. D. Ganji, “Approximate explicit solutions of nonlinear BBMB equations by He' s methods and comparison with the exact solution", Physics letters A, vol. 367, pp. 95-101, 2007"

[17] Ö. F. Gözükizil and Ş. Akçağil, “Exact solutions of Benjamin-Bona-Mahony-Burgers-type nonlinear pseudo-parabolic equations”, Boundary value problems, vol.144, pp. 01-12, 2012 